\title{
Photonic band gap of multiferroic-dielectric materials in the IR region: FDTD method
}

\author{
Selami Palaz ${ }^{\mathrm{a}}$, Sevket Simsek ${ }^{\mathrm{b}}$, Amirullah M. Mamedov ${ }^{\mathrm{c}, \mathrm{d}}$, and Ekmel Ozbay ${ }^{\mathrm{c}}$ \\ ${ }^{\mathrm{a}}$ Faculty of Science and Letter, Harran University, Urfa, Turkey; ${ }^{\mathrm{b}}$ Faculty of Engineering Department of \\ Material Science and Engineering, Hakkari University, Hakkari, Turkey; 'Bilkent University \\ Nanotechnology Research Center, Ankara, Turkey; ${ }^{d}$ Baku State University International Scientific Center, \\ Baku, Azerbaijan
}

\begin{abstract}
In this report, we present an investigation of the optical properties and band structure calculations for the photonic structures based on the multiferroic materials- $\mathrm{BaMnF}_{4}$. We calculate the photonic bands and optical properties of $\mathrm{BaMnF}_{4} / \mathrm{LiNbO}_{3}$ based photonic crystal. We study the photonic band gap and optical properties of the photonic structures, numerically analyzed in the IR frequency region by using the FDTD method for various incidence angles, number of periods in the PC and the nature/geometry of the materials.
\end{abstract}

\section{ARTICLE HISTORY}

Received 14 May 2018

Accepted 31 October 2018

\section{KEYWORDS}

Photonic crystal; $\mathrm{BaMnF}_{4} /$ $\mathrm{LiNbO}_{3}$; optical properties; multiferroic

\section{Introduction}

Photonic crystals (PCs) are structures with periodically modulated refractive indices whose distribution follows the periodicity of order of a fraction of the optical wavelength. They can generate spectral regions named stop (photonic) band gaps where light cannot propagate in a manner analogous to the formation of electronic band gaps in dielectrics. The properties of PCs strongly depend on the configuration of the selected materials, which cannot be modified after fabrication. In addition, the photonic band gap (PBG) structure depends on the refractive indices of the selected materials $[1,2]$. If the PBG structure of the PCs can be externally modulated by some other means, the PCs can be applicable as the active elements of many optoelectronic devices. Therefore, as the photonic crystalline material we used the $\mathrm{BaMnF}_{4} / \mathrm{LiNbO}_{3}$-system, which has interesting properties for the implementation of active PCs. This unique feature of the PCs one dimensional photonic crystal is attractive since it dramatically alters the flow of light and the manipulation of photons within the structure and their production is more feasible at any wavelength scale and their analytical and numerical calculations are simpler.

In this paper, we study the photonic band structure and optical properties of the $1 \mathrm{D}$ and $2 \mathrm{D} \mathrm{BaMnF}_{4} / \mathrm{LiNbO}_{3}$ based PCs with square lattice by using the FDTD technique, that is based on the computing eigenstates of Maxwell's equations for periodic systems $[3,4]$ 


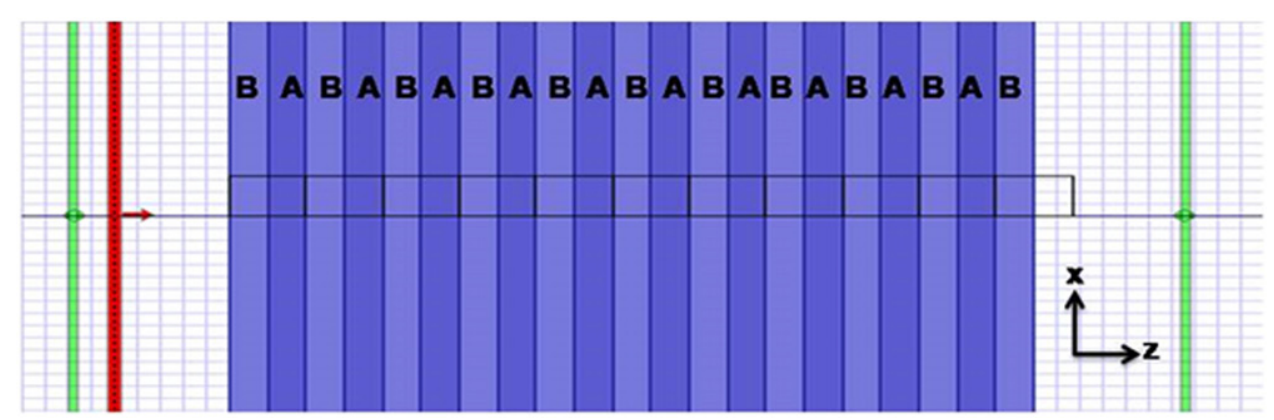

Figure 1. 1-Dimensional $\mathrm{BaMnF}_{4} / \mathrm{LiNbO}_{3}$ Based Photonic Crystal Structure (PCs).

\section{Computational details}

In our simulation, we employed the finite-difference time domain (FDTD) technique, which implies the solution of Maxwell equations with centered finite-difference expressions for the space and time derivatives. FDTD schemes are especially promising for the investigation of PBG structures, as they provide an opportunity for analyzing the spatial distribution of the electromagnetic field in PBG structure. All of the calculations in this work were performed using the OptiFDTD package [3].

It is well known that $\mathrm{LiNbO}_{3}$ and $\mathrm{BaMnF}_{4}$ are important material because of their nonlinear behavior. Here, a rectangle lattice with various types of scatterer in a dielectric background has been considered garding the effect of various parameters on a complete band gap has been investigated. The permittivity's of $\mathrm{BaMnF}_{4}$ and $\mathrm{LiNbO}_{3}$ were 3.80 and 5.52, respectively. Here $A$ and $B$ are defined as being two functional materials, with different refractive indices $\left(\mathrm{n}_{\mathrm{A}}, \mathrm{n}_{\mathrm{B}}\right)$ and they have geometrical layer thickness $\left(\mathrm{d}_{\mathrm{A}}, \mathrm{d}_{\mathrm{B}}\right)$. In place of materials $\mathrm{A}$ and $\mathrm{B}$, we used $\mathrm{BaMnF}_{4}$ (A) and $\mathrm{LiNbO}_{3}$ (B) [5-7]. In Fig. 1 we schematically show the geometry of conventional PCs. The BaMnF$/ 4 \mathrm{LiNbO}_{3}$ system is frequently used as special interest for the middle and deep infrared (IR) applications due to their high transmission in the IR region.

A typical 1D PCs is shown in Fig. 1. The thickness of the considered layers of $\mathrm{BaMnF}_{4}$ and $\mathrm{LiNbO}_{3}$ are $d_{\mathrm{LiNbO}_{3}}=0.4 a$ and $d_{\mathrm{BaMnF}_{4}}=0.6 a$, respectively. The lattice constant is $a=\left(d_{A}+d_{B}\right)=1 \mu m$. The filling fraction $\mathrm{f}$ is the ratio between the thickness of the lower refractive index layer (1.96) and the period of the PC, i.e., $f=d_{1} /\left(d_{1}+d_{2}\right)$. The filling fraction is set to 0.6. The refractive index contrast of $\mathrm{BaMnF}_{4}$ is 1.96. The refractive index of the background dielectric medium is assumed as $\mathrm{LiNbO}_{3}\left(\mathrm{n}_{\mathrm{LiNbO}_{3}}=2.35\right)$.

In our calculations, we used OptiFDTD software package [3]. The OptiFDTD software package is based on the FDTD method for transmission spectra and the plane wave expansion method (PWE) for photonic band structure.

The photonic band structures of the proposed PCs were calculated by solving the Maxwell equations. The FDTD algorithm is one of the most appropriate calculation tools [4]. For solving Maxwell's equations depending on the time, the FDTD algorithm divides space and time in a regular grid.

\section{Results and discussion}

We calculate the spectral propertiesup to the $n$-th generations $(n=10)$ periodic layered structures consisting of $\mathrm{BaMnF}_{4} / \mathrm{LiNbO}_{3}$ compounds. Band structure of $1 \mathrm{D}$ 


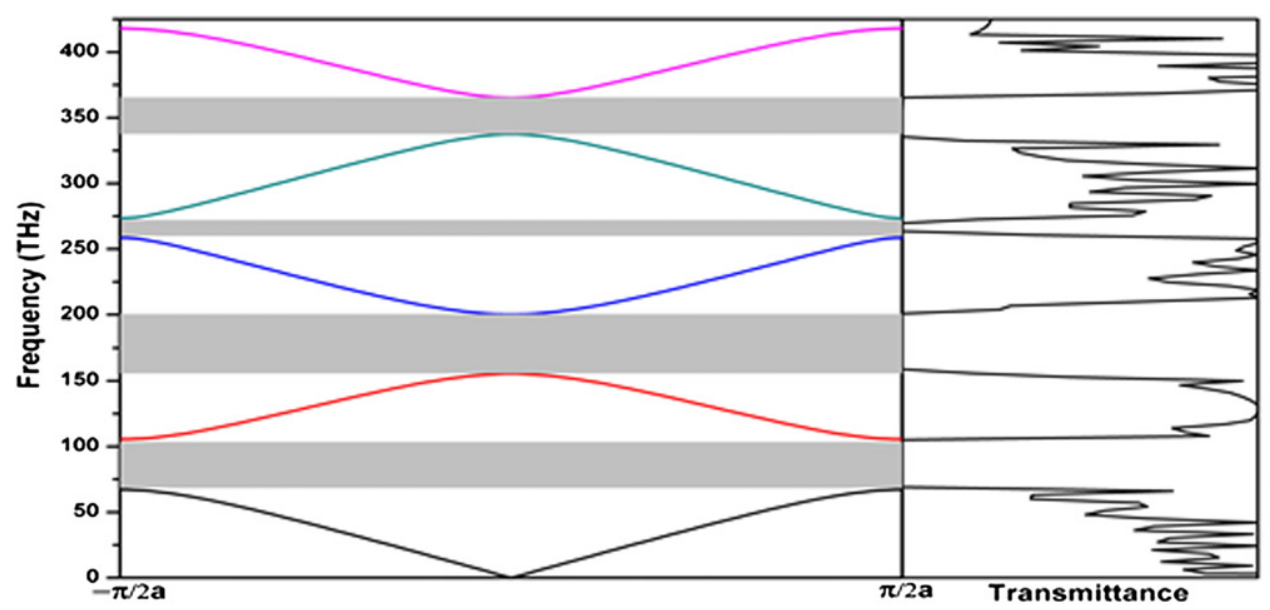

Figure 2. TE Band structure and transmittance spectra of $\mathrm{BaMnF}_{4} / \mathrm{LiNbO}_{3}$ in $1 \mathrm{D}$.

Table 1. Variation of full band gap size for TE modes with filling factor for anisotropic BaMnF 4 $\mathrm{LiNbO}_{3}$ based layers in air background

\begin{tabular}{|c|c|c|c|c|c|c|c|c|}
\hline \multirow[b]{2}{*}{$\begin{array}{l}\text { Filling } \\
\text { Factor }\end{array}$} & \multicolumn{2}{|c|}{ TE1 } & \multicolumn{2}{|c|}{ TE2 } & \multicolumn{2}{|c|}{ TE3 } & \multicolumn{2}{|c|}{ TE4 } \\
\hline & $\begin{array}{c}\text { Band } \\
\text { Gap (THz) }\end{array}$ & $\begin{array}{c}\text { Gap } \\
\text { Size (\%) }\end{array}$ & $\begin{array}{c}\text { Band } \\
\text { Gap (THz) }\end{array}$ & $\begin{array}{c}\text { Gap } \\
\text { Size (\%) }\end{array}$ & $\begin{array}{c}\text { Band } \\
\text { Gap (THz) }\end{array}$ & $\begin{array}{c}\text { Gap } \\
\text { Size (\%) }\end{array}$ & $\begin{array}{c}\text { Band } \\
\text { Gap (THz) }\end{array}$ & $\begin{array}{c}\text { Gap } \\
\text { Size (\%) }\end{array}$ \\
\hline 0.1 & $(101-148)$ & 37.75 & $(229-294)$ & 24.85 & $(365-432)$ & 16.81 & $(511-556)$ & 8.43 \\
\hline 0.2 & $(87-144)$ & 49.35 & $(213-260)$ & 19.87 & $(342-360)$ & 5.12 & $(438-501)$ & 13.41 \\
\hline 0.3 & $(77-134)$ & 54.02 & - & - & $(289-346)$ & 17.95 & - & - \\
\hline 0.4 & $(70-119)$ & 51.85 & (177-209) & 16.58 & $(274-301)$ & 9.39 & $(360-411)$ & 13.23 \\
\hline 0.5 & $(58-105)$ & 57.66 & $(155-200)$ & 25.35 & $(258-273)$ & 5.64 & (337-365) & 7.97 \\
\hline 0.6 & (64-93) & 37.94 & (140-183) & 26.62 & $(227-265)$ & 15.44 & (320-335) & 4.58 \\
\hline 0.7 & $(63-83)$ & 27.39 & $(131-165)$ & 22.97 & $(206-246)$ & 17.69 & $(286-323)$ & 12.15 \\
\hline 0.8 & $(62-75)$ & 18.97 & $(126-149)$ & 16.72 & $(193-224)$ & 14.86 & $(263-298)$ & 12.47 \\
\hline 0.9 & $(61-68)$ & 10.85 & $(124-136)$ & 9.23 & (187-204) & 8.69 & $(251-272)$ & 8.03 \\
\hline
\end{tabular}

$\mathrm{BaMnF}_{4} / \mathrm{LiNbO}_{3}$ based PCs have been calculated in high symmetry directions in the first Brillouin zone (BZ) and shown in Fig. 2. We can see that there are four photonic band gaps (PBG) in Fig. 2. The width of the PBG is (58-105) THz for the first band.

When the frequency of the incident electromagnetic wave drops in these PBGs, the electromagnetic wave will be reflected completely by the photonic crystal. It can be seen in Fig. 2, that the transmittance is zero in this range of frequencies where the refractive index of the structure is positive and the spectral width of the gaps is invariant with the change in the transmittance (Table 1).

The numerical results of variation of a full band gap with the changing filling factor from 0.1 up to 0.9 are given in Table 1 . The variation of band gap sizes (\%) as a function of filling factor changes between 5 and 58 for $\mathrm{BaMnF}_{4} / \mathrm{LiNbO}_{3}$. The largest gap size is approximately $58 \%$ when the filling factor is as high as 0.5 , but it decreases and the filling factor continues to increase.

On the other hand, the second, third and fourth band gap sizes do not change too much according to the filling factor, but it reaches the minimum values when the filling factor changes between 0.4 and 0.6 . 
Table 2. Variation of the full band gap size for TE modes with r/a for a 2D system

\begin{tabular}{|c|c|c|c|c|}
\hline \multirow[b]{3}{*}{ r/a } & \multicolumn{4}{|c|}{$\mathrm{BaMnF}_{4} / \mathrm{LiNbO}_{3}$} \\
\hline & \multicolumn{2}{|c|}{ TE1 } & \multicolumn{2}{|c|}{ TE2 } \\
\hline & Band Gap (THz) & Gap Size (\%) & Band Gap (THz) & Gap Size (\%) \\
\hline 0.10 & - & - & - & - \\
\hline 0.15 & $(133-146)$ & 9.31 & - & - \\
\hline 0.20 & $(116-139)$ & 18.03 & - & - \\
\hline 0.25 & $(105-132)$ & 22.78 & - & - \\
\hline 0.30 & $(98-115)$ & 15.96 & (171-189) & 10.00 \\
\hline 0.35 & $(90-103)$ & 13.47 & $(156-170)$ & 8.58 \\
\hline 0.40 & - & - & - & - \\
\hline 0.45 & - & - & - & - \\
\hline
\end{tabular}

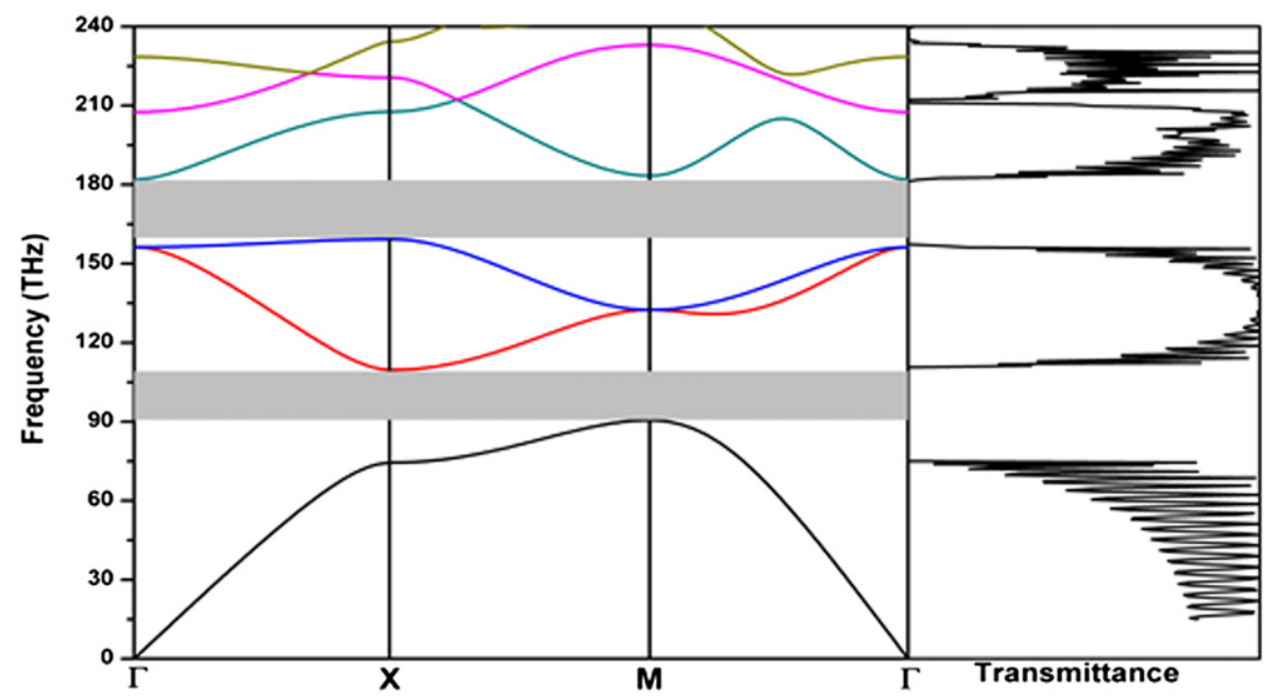

Figure 3. TE Band structures and transmittance spectra of $\mathrm{BaMnF}_{4} / \mathrm{LiNbO}_{3}$ in $2 \mathrm{D}$.

The transmittance spectra of $\mathrm{BaMnF}_{4} / \mathrm{LiNbO}_{3}$ based PCs of $\mathrm{n}$-th $(\mathrm{n}=5,6,7,8,9$, and 10) generations for the TE-polarized incident electromagnetic wave were also analyzed. The obtained transmission spectra show that the optical properties of the structure depend on the parity of $n$. The various spectra present bands of oscillations, one of which centered always in $\omega=165 \mathrm{THz}$. These bands narrow and the number of oscillations increase as $n$ increases. On the other hand, the positions of the minima in the transmission spectrum correlate with the gaps obtained in the calculation. Notably, the total number of transmission peaks is equal to the total number of elementary layers in the structure. This is a general property of multilayer structures. The transmission spectra of the $\mathrm{BaMnF}_{4} / \mathrm{LiNbO}_{3}$ structure also show scalability and that means the spectra of different generations have a similar shape when the frequency axis is properly scaled.

Higher-dimensional photonic crystals (2D) offer greater flexibility over 1D structures in the design of their geometry and potential applications. For example, several 1D crystals can be extended to 2D [7]. However, the design rules become increasingly complex as one considers the broader range of deterministic periodic or aperiodic structures. Along with the increase in geometrical complexity, there is a corresponding increase in 
the complexity of the mathematical description of the properties of electromagnetic propagation. Even without a detailed analytical description, however, good physical insight into the propagation properties of light can still be obtained in various photonic crystals. Therefore, we also calculate the band structure and optical properties for the same generations $\mathrm{BaMnF}_{4} / \mathrm{LiNbO}_{3}$ structures in $2 \mathrm{D}$.

In Fig. 3, we show the photonic band structures for the case of TE polarization for a square array of dielectric rods of $\mathrm{BaMnF}_{4} / \mathrm{LiNbO}_{3}$. The $r / a$ ratio is set to 0.3 . Where, $r$ is the radius of rods and $a$ is lattice constant $(a=1 \mu \mathrm{m})$. We can see that there are two PBGs in Fig. 3. The numerical results of variation of a full band gap for $\mathrm{BaMnF}_{4} /$ $\mathrm{LiNbO}_{3}$ based the 2D PCs are given in Table 2. The width of the PBG is (90-103) THz for the first band for the $\mathrm{BaMnF}_{4} / \mathrm{LiNbO}_{3}$.

\section{Conclusion}

The photonic band structures and transmission properties of the $1 \mathrm{D}$ and $2 \mathrm{D} \mathrm{BaMnF}_{4} /$ $\mathrm{LiNbO}_{3}$ based conventional PCs consisting of dielectric rods and layers $\left(\mathrm{BaMnF}_{4} /\right.$ $\mathrm{LiNbO}_{3}$ ) immersed in air were studied. The numerical results of the variation of the full band gap with a changing filling factor show that the largest gap size occurs when the filling factors are approximately 0.5 for $1 \mathrm{D}$. We have also investigated the spectral properties up to $n$-th generations $(n=10)$ of structures consisting of $\mathrm{BaMnF}_{4} / \mathrm{LiNbO}_{3}$ compounds. The obtained transmission spectra show that the optical properties of the structure depend on the parity of $n$.

\section{Funding}

This work is supported by the projects DPT-HAMIT. One of the authors (Ekmel Ozbay) also acknowledges partial support from the Turkish Academy of Sciences.

\section{References}

[1] S. V. Gaponenko, Introduction to Nanophotonics. (New York: Cambridge University Press, 2010).

[2] K. Sakoda, Optical Properties of Photonic Crystals (Berlin: Springer, 2005).

[3] OptiFDTD 10, http://www.optiwave.com/.

[4] A. Taflove, and S. C. Hagness, Computational Electrodynamics: The Finite-Difference Time-Domain Method 2nd ed. (Boston: Artech House Publishers, 2000).

[5] E. D. Palik, Handbook of Optical Constants of Solids (NY: Academic Press, 1998).

[6] A. Tressaud, and K. Poeppelmeier, Photonic and Electronic Properties of Fluoride Materials (Amsterdam: Elsevier, 2016).

[7] S. Simsek, A. M. Mamedov, and E. Ozbay, Two-dimensional ferroelectric photonic crystals: Optics and band structure. Ferroelectrics. 448(1), 23 (2013). 